\title{
ALCANCE TEÓRICO Y PRÁCTICO DEL TRANSNACIONALISMO: LA PARTICIPACIÓN POLÍTICA DE LOS INMIGRANTES NIGERIANOS EN LA CIUDAD DE NUEVA YORK
}

\author{
Theory and Practice of Transnationalism: The Political \\ Participation of Nigerian Immigrants in New York City
}

Leila Rodríguez ${ }^{1}$

\begin{abstract}
Resumen. El concepto de migración transnacional reconoce los lazos que los inmigrantes mantienen con sus países de origen. A pesar del gran número de estudios sobre los inmigrantes transnacionales, pocas investigadores han dedicado atención a las formas y mecanismos de participación simultánea en las comunidades de origen y recepción. En este artículo se analiza las motivaciones, mecanismos de y barreras a la participación política de los inmigrantes nigerianos en Nueva York y Nigeria. Basado en un estudio cualitativo con esta población, se concluye que el transnacionalismo político es un fenómeno que alcanza solamente una pequeña proporción de la comunidad de inmigrantes nigerianos, y que el acto de inmigrar altera la posición social relativa de los individuos con respecto a los nigerianos y estadounidenses, proporcionándoles los recursos necesarios para participar en Nigeria y limitando su involucramiento en EE.UU.
\end{abstract}

Palabras clave: inmigrantes nigerianos, transnacionalismo, migración transnacional, transmigrante, participación política.

\begin{abstract}
The concept of transnational migration recognizes the ties that migrants retain with their origin countries. Despite numerous studies about transmigrants, few researchers have dedicated attention to the forms and mechanisms of simultaneous participation in origin and reception communities. In this article I analyze the motivations, mechanisms of and barriers to transnational political participation of Nigerians in New York City and Nigeria. Based on a qualitative study with the migrant community, the article concludes that political transnationalism is limited to a small proportion of Nigerian immigrants, and that the act of migrating alters the relative social position of individuals with respect to Nigerians and Americans,
\end{abstract}

1 Departamento de Antropología University of Cincinnati. Cincinnati, EE.UU. 
providing the resources they need to participate in Nigeria but limiting their involvement in the United States.

Keywords: Nigerian immigrants, transnationalism, transnational migration, transmigrants, political participation.

\section{Introducción}

En las últimas décadas ha surgido desde la antropología y expandido a las demás ciencias sociales, el concepto del transnacionalismo como reconocimiento de los lazos que los inmigrantes mantienen con sus países de origen, y de las importantes consecuencias de esos lazos tanto para las comunidades de expulsión como las de recepción. La migración transnacional se refiere al proceso mediante el cual los inmigrantes forjan y sostienen relaciones simultáneas de múltiples hebras que unen a las unidades de origen y asentamiento ${ }^{2}$. La migración transnacional forma parte de un fenómeno más amplio de transnacionalismo, que incluye procesos económicos, políticos, y socioculturales que transcienden las fronteras de los estado-naciones, pero permanecen ligados a las oportunidades y limitaciones de sus contextos específicos $^{3}$. Este artículo repasa las teorías sobre la migración transnacional y re-evalúa el alcance de este fenómeno basándose en la experiencia de los inmigrantes nigerianos en la Ciudad de Nueva York. En particular, se analizan las condiciones bajo las cuales se facilita o impide el comportamiento transnacional de un grupo de inmigrantes, así como el alcance de sus actividades.

El transnacionalismo puede ocurrir en las esferas económicas, políticas y socioculturales ${ }^{4}$, y en distintos niveles de institucionalización $n^{5}$. Organizaciones No Gubernamentales que monitorean los derechos humanos a nivel mundial, activistas que organizan boicots de empresas para presionarlas a mejorar sus prácticas laborales en otros países, o partidos políticos que establecen sedes en otros países para atraer a los inmigrantes constituyen ejemplos de agentes transnacionales. Por este motivo, las actividades transnacionales se han conceptualizado por un lado, como un movimiento de resistencia "de abajo hacia arriba"6, donde personas marginalizadas emplean actividades transnacionales para resistir al estado u otras formas de dominio. Por otro lado,

2 GLICK-SCHILLER, Nina et alii. From Immigrant to Transmigrant: Theorizing Transnational Migration, p. 48.

3 GUARNIZO, Luis Eduardo, SMITH, Michael Peter. The Locations of Transnationalism.

4 PORTES, Alejandro. Introduction: The Debates and Significance of Immigrant Transnationalism.

5 PORTES, Alejandro et alii. The Study of Transnationalism: Pitfalls and Promise of an Emergent Research Field.

6 GUARNIZO, Luis Eduardo. On the Political Participation of Transnational Migrants: Old Practices and New Trends; PORTES, Alejandro. Conclusion: Theoretical Convergences and Empirical Evidence in the Study of Immigrant Transnationalism. 
algunas prácticas transnacionales también pueden ser vistas como herramientas que permiten al estado ejercer poder fuera de su jurisdicción transnacional ${ }^{7}$.

Los inmigrantes constituyen un ejemplo de transnacionalismo de abajo hacia arriba. Glick-Schiller, Basch y Szanton Blanc, fueron las primeras en acuñar el término "transmigrante" en 1995 para referirse a estos inmigrantes que mantienen lazos en al menos dos países, y delinearon tres causas de la existencia de estos transmigrantes ${ }^{8}$ : la restructuración global del capital que ha deteriorado las condiciones sociales y económicas en los países de envío y recepción de inmigrantes, el auge del racismo en los contextos de recepción, y los proyectos de construcción de la nación en ambos países de envío y recepción que construyen lealtades hacia ambos entre los inmigrantes.

A pesar de la anticipación inicial sobre este fenómeno, los debates al respecto pronto se centraron en cuatro temas. Primero, se debatió la cobertura de este fenómeno pues en la literatura temprana se refería a él como omnipresente. Portes argumenta que este sobre énfasis se dio en parte porque los primeros estudios al respecto fueron cualitativos y por ende se enfocaron solamente en aquellas personas que sí eran transmigrantes ${ }^{9}$. Estudios cuantitativos posteriores demostraron que el fenómeno es más limitado de lo que al principio se pensaba. Segundo, se cuestionó si realmente la migración transnacional constituía un nuevo fenómeno digno de ser teorizado, pues todas las olas de inmigrantes previas han mantenido algún tipo de lazo con sus países de origen ${ }^{10}$. Tercero, se cuestionó la falta de una definición única sobre el transmigrante, ya que muchos tipos de migraciones recibieron ese término ${ }^{11}$. Finalmente, se exigió mayor atención a la significancia del fenómeno, y en sus consecuencias ${ }^{12}$. Se ha llegado a algunas convergencias sobre el tema: la migración trasnacional no constituye un nuevo fenómeno sino una nueva perspectiva, el alcance y las formas de transnacionalismo varían según los contextos de envío y recepción, y a pesar de que no todos los inmigrantes son transnacionales, sus actividades tienen consecuencias macrosociales potencialmente de gran importancia ${ }^{13}$. Los esfuerzos académicos más recientes se han concentrado en estudiar la relación entre integración en el país receptor y la participación en actividades transnacionales ${ }^{14}$, el reconocimiento de compromisos tanto con los países de

7 GLICK-SCHILLER, Nina. Transmigrants and Nation-States: Something Old and Something New in U.S. Immigrant Experience.

8 GLICK-SCHILLER, From Immigrant..., op. cit.

9 PORTES, Introduction..., op. cit.

10 Ibidem.

11 Ibidem.

12 Ibidem.

${ }^{13}$ IDEM. Conclusion: Theoretical Convergences and Empirical Evidence in the Study of Immigrant Transnationalism.

${ }^{14}$ ERDAL, Marta Bivand, OEPPER, Ceri. Migrant Balancing Acts: Understanding the Interactions 
origen como de recepción ${ }^{15}$, y la identificación de los mecanismos que generan y alterar las interacciones transnacionales ${ }^{16}$. Este artículo lidia con este último tema, y analiza los mecanismos que facilitan o impiden las actividades políticas transnacionales de los inmigrantes nigerianos en la Ciudad de Nueva York, así como el alcance de sus actividades.

\section{Diseño y Métodos de Investigación}

Los datos de este artículo provienen de un año de trabajo de campo en la Ciudad de Nueva York que incluyó la realización de treinta entrevistas cualitativas con inmigrantes nigerianos así como doce entrevistas con líderes de distintas organizaciones de su comunidad ${ }^{17}$. En total, localicé 40 organizaciones de nigerianos, incluyendo tres profesionales (abogados, enfermeros y trabajadores sociales), seis de interés común (fútbol, jóvenes, etc.), y 31 basadas en etnicidad o comunidad de origen. Las entrevistas con los líderes de las organizaciones indagaron sobre las metas de estos grupos, su establecimiento y financiamiento, y obstáculos en sus operaciones. Las entrevistas con los inmigrantes recopilaron información demográfica, educacional, migratoria, familiar, laboral, e incluyeron preguntas acerca de su participación en diversas actividades políticas en ambos Nigeria y los Estados Unidos. Cabe recalcar que aunque en el proyecto se entrevistaron tanto hombres como mujeres, los principales actores políticos son todos hombres y esto es reflejado en los fragmentos de las entrevistas citadas aquí.

\section{Inmigración de Nigerianos a los Estados Unidos}

La migración contemporánea de africanos a los Estados Unidos nace de una combinación de factores que incluyen cambios en las economías africanas y en las leyes de inmigración estadounidenses. En África, problemas económicos de la post-independencia llegaron a un punto máximo en los 1970, y la inseguridad alimentaria, alta inflación, y desempleo afectaron a muchos países ${ }^{18}$. La introducción de los Programas de Ajuste Estructural en los 1980 impulsó mayores cambios en los sistemas económicos de control estatal a privatización, devaluó las monedas locales, y obligó a los gobiernos a reducir los

Between Integration and Transnationalism; ITZIGSOHN, José, SAUCEDO, Silvia Giorguli. Immigrant Incorporation and Sociocultural Transnationalism.

${ }^{15}$ BERMUDEZ, Anastasia. The Transnational Political Practices of Colombians in Spain and the United Kingdom: Politics 'here' and 'there'; MORAWSKA, Ewa. Immigrant Transnationalism and Assimilation: A Variety of Combinations and the Analytic Strategy it Suggests.

${ }^{16}$ WALDINGER, Roger. Immigrant Transnationalism.

17 El proyecto fue financiado por la Fundación Nacional de la Ciencia, EE.UU. (BCS-0718968).

${ }^{18}$ TAKYI, Baffour, KONADU-AGYEMANG, Kwadwo. Theoretical Perspectives on African Migration. 
programas de ayuda social ${ }^{19}$. Estas condiciones de deterioro socioeconómico crearon los incentivos para la emigración.

En los Estados Unidos, el Acto de Inmigración y Naturalización de 1965 alteró los criterios bajo los cuales se admitían inmigrantes al país, eliminando cuotas que favorecían la inmigración de europeos, y valorando la reunificación familiar como la principal condición para admitir nuevos inmigrantes ${ }^{20}$. En 1990 también se introdujo el programa de Lotería de Visas de Diversidad que aumentó la migración de africanos al país. De todos los países elegibles, los nigerianos han obtenido el mayor número de estas visas desde el 2002, aproximadamente el $14 \%$ de las 50.000 anuales $^{21}$.

Actualmente hay más de 1,5 millones de africanos en los Estados Unidos ${ }^{22}$. Los inmigrantes africanos tienen altos niveles de educación, y más del 90\% terminaron la educación secundaria. Entre los inmigrantes, tienen las tasas más altas de participación laboral y menor probabilidad de ser desempleados, pero menor probabilidad de haberse naturalizado ${ }^{23}$. Los nigerianos conforman el grupo más grande de inmigrantes africanos en los Estados Unidos, aproximadamente el $14 \%{ }^{24}$.

Nigeria es conformado por unos 250 grupos étnicos distintos, de los cuales los tres mayores son los Ibo, Yoruba, y Hausa-Fulani. Aunque no existen estimaciones de qué porcentaje de los nigerianos en los Estados Unidos provienen de estos grupos, las personas entrevistadas para este estudio mencionaron que las concentraciones más grandes son los Ibo, Yoruba, y algunos grupos minoritarios como los Edo y Bini. La población de inmigrantes nigerianos en los Estados Unidos sigue en aumento, son altamente educados, hablan inglés, y participan extensamente en asociaciones comunitarias basadas en su étnica y comunidad de origen.

\section{Resultados}

Los inmigrantes pueden participar en la esfera política de sus países de origen y recepción de múltiples formas. Cuando se han naturalizado pueden votar en elecciones políticas de sus países de recepción, pueden participar en actos y manifestaciones políticas organizadas por asociaciones comunales, y pueden involucrarse en la política de sus países de origen votando en las elecciones, haciendo campaña para algún candidato, o realizando críticas

\footnotetext{
19 Ibidem.

20 ARTHUR, John. Invisible Sojourners: African Immigrant Diaspora in the United States.

21 UNITED STATES DEPARTMENT OF STATE. Cf. < http://travel.state.gov>.

22 MCCABE, Kristen. African Immigrants in the United States.

${ }^{23}$ DIXON, David. Characteristics of the African Born in the United States.

24 MCCABE, op. cit.
} 
de gobernantes actuales ${ }^{25}$. En mi estudio, encontré que los inmigrantes nigerianos participan en múltiples actividades transnacionales. Algunas de éstas, como el emprendimiento transnacional ${ }^{26}$ y el envío de remesas familiares son principalmente económicas y no serán discutidas en este artículo. Otras actividades son de carácter político, incluyendo el seguimiento de acontecimientos políticos en ambos países, votar en elecciones, y participar en organizaciones comunales que organizan actividades políticas.

Las asociaciones nigerianas en Nueva York varían en tamaño y alcance. Algunas, en particular las que representan a los grupos étnicos mayoritarios, tienen cientos de miembros y son capaces de realizar múltiples grandes proyectos. Otras, especialmente las asociaciones comunales de grupos minoritarios, son mucho más pequeñas y llevan a cabo proyectos más pequeños. Las asociaciones grandes tienen múltiples capítulos a través de los Estados Unidos, organizan una convención anual. Como describió Emmanuel27, miembro de una de las mayores asociaciones:

Yo vengo de la tribu Yoruba y pertenezco al capítulo de yorubas en Nueva York. Es una asociación que provee una plataforma para que los yoruba nos unamos y veamos que podemos hacer por nuestros hijos y nuestra cultura, y preservar los ideales de nuestra tradición. Organizamos eventos como ceremonias de graduación para los estudiantes, tenemos programas de becas para ayudar a los niños en Nigeria, y organizamos el Día Yoruba para promover nuestra cultura a los demás.

Muchos de los entrevistados concordaron con lo expresado por Emmanuel. Aunque las asociaciones son auto catalogadas como "culturales", sus actividades con frecuencia cruzan al ámbito político. Las asociaciones nigerianas realizan actividades tanto en Nigeria como los Estados Unidos. Las principales actividades en Nigeria involucran proyectos filantrópicos, como las becas para que los jóvenes puedan asistir a la universidad, donación de equipo hospitalario, realizar campañas de vacunación, y reparar las carreteras de las comunidades de origen. Estas actividades con frecuencia requieren la coordinación con las autoridades gubernamentales locales o estatales de Nigeria, uniendo de distintas maneras a los miembros de las asociaciones a la estructura política. Edegbe, miembro de otra grande asociación, comentó:

Trabajamos de mano con el gobierno de nuestro estado. Y cada año introducimos un par de programas. Tuvimos una misión médica, la

\footnotetext{
${ }^{25}$ GUARNIZO, On the Political..., op. cit.; LEVITT, Peggy, JAWORSKY, Nadya. Transnational Migration Studies: Past Developments and Future Trends; RICHMAN, Karen. "Call Us Vote People": Citizenship, Migration, and Transnational Politics in Haitian and Mexican Locations.

${ }^{26}$ Ver RODRIGUEZ, Leila. Maximizing Social Proximity in Market Relations: The Networks of Nigerian Immigrant Business Owners in New York City.

27 Todos los nombres utilizados son pseudónimos.
} 
tenemos cada año. Va un grupo de médicos y enfermeras y tratamos a los pacientes gratuitamente. También tenemos una misión educativa para dar becas. Y nos importa mucho la buena gobernación. Ponemos mucha atención a cómo el gobierno trabaja. Y mandamos a miembros de nuestro grupo a ver como están gobernando, y si vemos algo que no nos gusta somos muy críticos. Hacemos un reporte. Y el gobierno estatal está consciente de nosotros, y trabajan con nosotros.

El comentario de Edegbe sugiere al menos dos formas en las que las asociaciones de nigerianos en los Estados Unidos se involucran políticamente en Nigeria. Primero, los proyectos diseñados para desarrollar las comunidades locales -becas educativas, misiones médicas, construcción de carreteras, etc.-, son coordinados con las autoridades locales. De hecho, algunos de los proyectos cumplen labores que deben en principio ser realizadas por las autoridades. De esta forma, la labor de los migrantes llena algunos de los vacíos en la gobernación oficial de las comunidades. Segundo, algunas asociaciones también actúan como monitores de los gobiernos estatales y locales, un papel que en ocasiones ha llevado a conflictos. Las entrevistas revelaron importantes patrones en las motivaciones, mecanismos de y barreras a la participación política transnacional de las asociaciones y sus miembros.

\section{Motivaciones}

Aunque la mayoría de las asociaciones nigerianas en Nueva York ayudan a sus comunidades de origen, algunas fueron creadas específicamente para ayudar a los inmigrantes nigerianos en los Estados Unidos. Una de esas asociaciones es dirigida por una sola persona que conoce una red de abogados, doctores y pastores dispuestos a proveer servicios, consejos y alojamiento temporal gratuitamente a los nigerianos que no pueden localizar a sus familiares al llegar a Nueva York. Parte de su motivación es que muchos compatriotas piden ayuda en el consulado nigeriano pero no la reciben. El director de este grupo recontó:

Solamente hoy ya he recibido tres llamadas. Uno es de un nigeriano que está de visita, tiene un hijo enfermo de anemia falciforme, y quiere saber cómo le podemos ayudar. Pues no le podemos ayudar porque no tenemos los recursos para ayudarle. Y el consulado de Nigeria? No, el consulado no puede ayudar porque no tienen fondos para eso. A ver, qué podemos hacer? Bueno, podemos hacer una cosa por ellos, y es encontrarles un lugar donde hospedarse.

Tanto las asociaciones que se enfocan en los inmigrantes como las que ayudan a sus comunidades de origen en Nigeria trabajan para llenar lo que sus líderes consideran como un vacío dejado por las autoridades nigerianas, tanto en Nigeria como en su representación diplomática en el extranjero. Este papel se ha desarrollado con el paso del tiempo, ya que todas las asociaciones 
al inicio fueron establecidas para ayudar a los inmigrantes a aclimatarse a su nuevo hogar. Otro entrevistado explicó:

Cuando migramos los primeros no teníamos ningún cuerpo constituido como guía. Cualquier dirección era de uno a uno, algún amigo o conocido. Pero formamos esta asociación para que nos vieran como una sociedad africana, un grupo auténtico de profesionales dedicados a las causas de los africanos. En ese tiempo era los 80, no podíamos identificar algo similar. Eso nos motivó a establecer el grupo.

Otra forma de participación política en los Estados Unidos es el voto. Evidentemente, sólo los nigerianos que se han naturalizado como ciudadanos de los Estados Unidos pueden votar en las elecciones generales (aunque algunas localidades permiten a todos sus residentes votar en elecciones menores) y no todos los que pueden votar lo hacen. No obstante, los entrevistados que sí votan dieron crédito a lo que perciben como transparencia en el proceso electoral estadounidense como una motivación para votar. Los periódicos locales de la comunidad de inmigrantes nigerianos también incitan a los inmigrantes a votar. Un editorial incitaba a los inmigrantes naturalizados a votar porque "los inmigrantes han sido delegados al estado de ciudadanos de tercera clase en [Estados Unidos] y para revertir esto los inmigrantes deben manifestarse en voz alta en la próxima elección presidencial"28. Otro periódico recordó a los nigerianos que aunque sus experiencias en las elecciones nigerianas probablemente han sido negativas, dado que son manipuladas, esto no debería desmotivarlos a votar en las elecciones estadounidenses ${ }^{29}$.

Las asociaciones dirigidas hacia sus comunidades de origen mencionaron las necesidades de los pobladores como su mayor motivación para participar en ellas. Un entrevistado explicó que el primer gobernador de su estado después del fin de los gobiernos militares en Nigeria era corrupto e ineficiente:

Las escuelas estuvieron cerradas un año entero. La infraestructura estaba mal, las carreteras estaban en mal estado, los hospitales empleaban doctores y enfermeras que no estaban debidamente acreditados. Todo realmente estaba terriblemente mal. Nada servía. Y eso fue lo que nos hizo crear nuestra asociación. Porque nuestras familias nos pedían ayuda.

Aunque pocas personas lo mencionaron, algunos miembros de las asociaciones, en particular los líderes han usado sus labores en Nigeria para aumentar su influencia política en sus comunidades de origen, y algunos pretenden regresar a gobernar ellos mismos. Un entrevistado sí admitió que "tratamos de expandirnos y buscar un cargo público".

28 EDITORIAL. Immigrants' Role in the 2008 Election.

${ }^{29}$ EDITORIAL. Why You Must Vote. 
En resumen las motivaciones para participar políticamente en Nigeria son numerosas. Varían desde el beneficio individual (como lanzar candidaturas políticas en Nigeria), beneficio grupal (promover la comunidad nigeriana en Nueva York), a altruistas (alivianar los problemas enfrentados por sus familiares y comunidades en Nigeria). Las motivaciones para participar en los Estados Unidos, sin embargo, son menores y son promovidas por algunos líderes comunales y medios de comunicación de la comunidad inmigrante.

\title{
Mecanismos
}

Para los inmigrantes nigerianos, vivir en el extranjero es el principal factor que les permite participar políticamente en su país de origen. Quienes viven en los Estados Unidos, aún teniendo salarios modestos, usualmente están en mejores condiciones financieras que cuando residían en sus comunidades de origen, y utilizan esa posición ventajosa para ejercer influencia política. Como explicó un entrevistado:

\begin{abstract}
Yo voy al gobierno local [nigeriano] y les digo, vean, yo gano tanto dinero. Puedo hacer contribuciones a su campaña, así que cuide de mí y de las cosas que me importan. Ahora, yo puedo hacer eso porque vivo aquí en los Estados Unidos. Si gano mucho dinero o gano la lotería puedo ir a mi gobierno y darle la mitad. No se olvide que todo tiene que ver con el estatus social de todo el mundo. Su estatus social lo determina el poder. Y qué es el poder? Puedes tener mucho dinero y comprar todo.
\end{abstract}

Esta cita revela otro patrón en las entrevistas: aquellas personas que reportan mayores niveles de educación y salariales participaban en mayor grado en las asociaciones nigerianas. Aunque muchos de los entrevistados discutieron el poder del dinero, evidentemente no todos los inmigrantes nigerianos son suficientemente adinerados para ejercer tal grado de influencia en la política local nigeriana. No obstante, la importancia de ser relativamente adinero por motivo de residir y trabajar en los Estados Unidos fue repetida en múltiples entrevistas. Para Akinlabi, inmigrante de clase media, el dinero y las remesas familiares también aumentan el poder político de los familiares que permanecen en las comunidades de origen.

La gente aquí tiene más dinero que la gente allá. Entonces la gente aquí tiene una ventaja, y qué podemos hacer? Las cosas cambian, cuando la gente viene a Estados Unidos su estilo de vida cambia. No olvide también, venimos a mejorar nuestras vidas. Entonces cuando llegas, realmente quieres mejorar tu vida, no vas a regresar. Y una de las cosas que hasta el gobernador [en Nigeria] está descubriendo es que ahora hay gente aquí preguntando por qué está haciendo esto el gobernador. Están buscando a las personas que envían remesas porque nuestros familiares dependen de que los de aquí enviemos dinero. Usted se podrá preguntar, por qué le importa esto al gobernador? Porque como te dirán los de aquí, 
cuando envío dinero a mis familiares les digo a quien apoyar. Entonces el gobernador tiene que convencer a algunas personas de aquí a que lo apoyen, y los de aquí tenemos que decidir a quien apoyar y decirle a nuestros familiares a quien apoyar. Sí, los de aquí realmente se involucran.

La importancia de la comunidad inmigrante nigeriana es evidente en su relación con los líderes de sus comunidades y estados de origen. Los inmigrantes no sólo son cortejados durante las campañas electorales, pero los gobernadores viajan a los Estados Unidos a reunirse con las asociaciones más grandes y participar en sus convenciones anuales. Quienes se involucran políticamente aprovechan la oportunidad de esas visitas para ejercer influencia sobre el gobierno en Nigeria. Un entrevistado comentó: "Hasta el actual gobernador del estado no ha podido gobernar bien. Ahora, él sabe cuáles son los problemas, y estuvo aquí en Nueva York hace como un mes, entonces le señalamos la educación y otras áreas que necesitan mejorar".

Además del estatus social conferido por el dinero, vivir en el extranjero también le ofrece a los inmigrantes la libertad de confrontar a los gobiernos locales nigerianos sin miedo a repercusiones en su contra. Uzo fue presidente de una asociación de nigerianos en Nueva York. Su asociación, que reúne a personas originarias del sureste de Nigeria, tuve el papel político más directo que no involucró el apoyo a un partido o candidato específico, sino más bien un compromiso con la lucha contra la corrupción. Una meta de su asociación es asegurar la buena gobernación de su estado de origen. Él detalló un evento específico en el que los líderes de la asociación confrontador a un gobernador y evitaron que se re-lanzara como candidato en las siguientes elecciones.

No había nadie que pudiera criticar al gobierno, nadie que podía tomar una posición neutral. Todos querían quedar bien con el gobierno entonces necesitábamos una voz externa independiente. Como estamos en los Estados Unidos y no dependemos del gobierno de Nigeria, no nos pagan, no nos mantienen, no necesitamos su dinero, somos profesionales aquí entonces es más fácil para nosotros tomar posiciones independientes. Había mucho conflicto entonces yo fui a Nigeria al visitar la oficina del gobernador, y al final del día, después de discutir con él salió forzosamente de su oficina y dijo que iba a alguna parte, que no hablaría más con nosotros. Entonces fuimos a la prensa y lo denunciamos y después de que hicimos eso, eso le dio valor a las personas. Todos empezaron a criticarlo y eventualmente así cayó, cuando hubo otra elección no pude lanzarse como candidato porque la gente ya lo había condenado.

Después de un periodo conflictivo con el gobierno que acusaron de corrupción, miembros de esa asociación ahora son llamados como observadores internacionales de las elecciones locales, y reciben a los líderes de su comunidad de origen en su convención nacional en los Estados Unidos. La labor de esta asociación resalta entre las demás: exige que el gobierno realice 
sus obligaciones y no crea dependencia a largo plazo en donde los inmigrantes se responsabilicen por suplementar el trabajo del gobierno nigeriano.

\section{Barreras}

Los inmigrantes nigerianos también enfrentan algunas barreras que impiden o limitan la actividad política transnacional. Quienes quieren participar en Nigeria, deben poder pagar el pasaje aéreo, un gasto elevado. La corrupción y decepción en el sistema electoral también impiden la participación. Como mencionó un entrevistado, "Hay algunos líderes que se portan mal y traicionan la confianza de la gente".

Algunas asociaciones que sí viajan a Nigeria evitan recaudar muchos fondos públicos o llevar a cabo ciertos proyectos por miedo a ser vistos como muy partidista: "Tenemos cuidado de no hacer demasiadas cosas para que sigamos siendo vistos como independientes, y podamos continuar siendo una voz política constructiva. En otras palabras, hay algunas cosas que no hacemos para que no se convierta en algo demasiado político. Tenemos que encontrar un balance".

Otras asociaciones deben lidiar con problemas internos, en particular miembros que no pagan su membresía o malos líderes. Incluso en asociaciones grandes, para la mayoría la participación se limita a pagar la membresía y atender las reuniones y eventos. Esto recalca el hecho de que el transnacionalismo político requiere de ciertos recursos y posiciones sociales dentro de la comunidad migratoria que no todos los individuos poseen.

Por otra parte, la participación en los Estados Unidos es limitada principalmente por falta de residencia permanente o ciudadanía. Esta situación podría cambiar mientras los nigerianos continúen recibiendo un gran porcentaje de visas de diversidad y migren como residentes permanentes. Algunos de los entrevistados mencionaron que el ser negros es un obstáculo adicional que enfrentan en los Estados Unidos que no enfrentaban en Nigeria: "Nuestra motivación de alguna forma no puede adaptarse a una sociedad complicada como los Estados Unidos, especialmente Nueva York. Es difícil, seas dominicano, hispano, africano o afro-americano. Si eres negro no hay ninguna diferencia, todos te ven como 'negro'".

\section{Conclusiones}

Las críticas tempranas al concepto de transmigrante argumentaron que no todos los inmigrantes son transmigrantes, y que se necesitan más investigaciones empíricas para entender el alcance y las formas de este fenómeno. También se ha recalcado la necesidad de analizar conjuntamente las actividades que los inmigrantes realizan simultáneamente en sus países de origen y recepción. En este artículo he tomado ese reto, y he analizado las motivaciones, mecanismos 
de y barreras a la participación política transnacional de los inmigrantes nigerianos tanto en los Estados Unidos como en su país de origen.

Este estudio confirma que no todos los inmigrantes internacionales son transnacionales, pues no todos realizan actividades económicas, políticas o socioculturales simultáneamente en sus comunidades de origen y de recepción. El enfoque de este artículo son las actividades políticas transnacionales, y aunque el estudio cualitativo no midió el alcance de este fenómeno, las entrevistas revelan que es un grupo pequeño de inmigrantes nigerianos que exhiben comportamientos políticos transnacionales. Quienes sí son actores políticos transnacionales son casi exclusivamente hombres, con muchos años de haber inmigrado, y con un estatus social importante dentro de la comunidad inmigrante. Las actividades transnacionales requieren de muchos recursos: sociales, financieros, humanos, y demás, y es evidente que no todos los inmigrantes cuentan con dichos recursos.

El acto de migrar a los Estados Unidos es un mecanismo que por sí solo altera la posición relativa de los individuos nigerianos. En los Estados Unidos, su condición de inmigrantes los coloca en una posición social relativamente inferior al de los ciudadanos, y por su tez son catalogados, junto con otras poblaciones de afro descendientes, como "negros", una categoría social por la cual también sufren discriminación. Sin embargo, a la vez que como inmigrantes se encuentran en una posición social inferior en los Estados Unidos, en Nigeria más bien suben de categoría social en sus comunidades de origen por residir en los Estados Unidos. Este cambio simultáneo en su posicionamiento social explica por qué su participación política es mayor en Nigeria que en los Estados Unidos.

Por último, el artículo recalca que si bien la migración transnacional no constituye un nuevo fenómeno (mas si una nueva perspectiva teórica), y no es un fenómeno que alcance a toda la población inmigrante, es un proceso social que tiene consecuencias macrosociales potencialmente muy altas. En el caso de los inmigrantes nigerianos, sus actividades como miembros de organizaciones comunales, en particular, han cambiado la forma de hacer política y de gobernación en muchas comunidades de Nigeria.

\section{Referencias}

ARTHUR, John. Invisible Sojourners: African Immigrant Diaspora in the United States. Westport: Praeger, 2000.

BERMUDEZ, Anastasia. The Transnational Political Practices of Colombians in Spain and the United Kingdom: Politics 'here' and 'there'. Ethnic and Racial Studies, v. 33, n. 1, 2010, p. 75-91.

DIXON, David. Characteristics of the African Born in the United States. Migration Information Source, 2006. Disponible en: <http://www.migrationinformation. org/USFocus/display.cfm?ID=366>. 
EDITORIAL. Immigrants' Role in the 2008 Election. U.S. Immigration News. 2:23, 2008.

EDITORIAL. Why You Must Vote. Light of the World. 5:62, 2008.

ERDAL, Marta Bivand; OEPPER, Ceri. Migrant balancing acts: understanding the interactions between integration and transnationalism. Journal of Ethnic and Migration Studies, v. 39, n. 6, 2013, p. 867-884.

GLICK-SCHILLER, Nina; BASCH, Linda; SZANTON, B. Cristina. From Immigrant to Transmigrant: Theorizing Transnational Migration. Anthropology Quarterly, v. 68, n. 1, 1995, p. 48-63.

GLICK-SCHILLER, Nina. Transmigrants and Nation-States: Something Old and Something New in U.S. Immigrant Experience. In HIRSCHMAN, Charles; DE WIND, Josh; KASINITZ, Philip (eds.). Handbook of International Migration: The American Experience. New York: Russell Sage, 1999, p. 94-119.

GUARNIZO, Luis Eduardo. On the Political Participation of Transnational Migrants: Old Practices and New Trends. In GERSTLE, Gary; MOLLENKOPF, John (eds.). E Pluribus Unum? Contemporary and Historical Perspectives on Immigrant Political Incorporation. New York: Russell Sage Foundation, 2001, p. 213-263.

GUARNIZO, Luis Eduardo; SMITH, Michael Peter. The Locations of Transnationalism. In SMITH, Michael Peter; GUARNIZO, Luis Eduardo (eds.). Transnationalism from Below. Comparative Urban and Community Research, v. 6. New Brunswick: Transaction Publishers, 1998, p.213-263.

ITZIGSOHN, José; SAUCEDO, Giorguli. Immigrant Incorporation and Sociocultural Transnationalism. International Migration Review, v. 36, n. 3, 2002, p. 766-798.

LEVITT, Peggy; JAWORKSKY, Nina. Transnational Migration Studies: Past Developments and Future Trends. Annual Review of Sociology, v. 33, p. 129156, 2007

MCCABE, Kristen. African Immigrants in the United States. Migration Information Source, 2011. Disponible en: <http://www.migrationpolicy.org/article/africanimmigrants-united-states $>$.

MORAWSKA, Ewa. Immigrant transnationalism and assimilation: a variety of combinations and the analytic strategy it suggests. In JOPPKE, Christian; MORAWSKA, Ewa (eds.). Toward assimilation and citizenship: Immigrants in liberal nation-states. United Kingdom: Palgrave Macmillan, 2014, p. 133-176.

PORTES, Alejandro. Introduction: The Debates and Significance of Immigrant Transnationalism. Global Networks, v. 1, n. 3, 2001, p. 181-193.

PORTES, Alejandro. Conclusion: Theoretical Convergences and Empirical Evidence in the Study of Immigrant Transnationalism. International Migration Review, v. 37, n. 3, 2003, p. 874-892.

PORTES Alejandro; GUARNIZO, Luis Eduardo; LANDHOLT, Patricia. The Study of Transnationalism: Pitfalls and Promise of an Emergent Research Field. Ethnic and Racial Studies, v. 22, n. 2, 2009, p. 217-237. 
RICHMAN, Karen. "Call Us Vote People": Citizenship, Migration, and Transnational Politics in Haitian and Mexican Locations. In REED-DANAHAY, Deborah; BRETTELL, Caroline B. (eds.). Citizenship, Political Engagement, and Belonging: Immigrants in Europe and the United States. New Brunswick: Rutgers University Press, 2008, p. 162-180.

RODRIGUEZ, Leila. Las Prácticas Empresariales de los Inmigrantes Nigerianos en Nueva York: Estructura, Agencia y Cambio y Continuación Cultural. Comunicación, Cultura y Política. Revista de Ciencias Sociales, v. 4, n. 1, 2013, p. 127-145.

RODRIGUEZ, Leila. Maximizing Social Proximity in Market Relations: The Networks of Nigerian Immigrant Business Owners in New York City. Research in Economic Anthropology, v. 36, 2016, p. 117-140.

TAKYI, Baffour; KONADU-AGYEMANG, Kwadwo. Theoretical Perspectives on African Migration. In KONADU-AGYEMANG, Kwadwo; TAKYI, Baffour; ARTHUR, John A. (eds.). The New African Diaspora in North America: Trends, Community Building and Adaptation. Lanham: Lexington Books, 2006, p. 13-28.

UNITED STATES DEPARTMENT OF STATE. Disponible en: < http://travel.state. gov>.

WALDINGER, Roger. Immigrant transnationalism. Current Sociology, v. 61, n. 5-6, 2013, p. 756-777.

Recibido para publicación en 16.07.2016

Aceptado para publicación en 23.08.2016

Received for publication in July $16^{\text {th }}, 2016$

Accepted for publication in August 23 $3^{\text {rd }}, 2016$

ISSN impresso 1980-8585

ISSN eletrônico 2237-9843

http://dx.doi.org/10.1590/1980-85852503880004704 